\title{
Patterns and persistence of behavioural and psychological symptoms in those with cognitive impairment: the importance of apathy
}

Running head: The course of behavioural and psychological symptoms

Key words: Behavioural and Psychological Symptoms; cognitive impairment; apathy; cohort study

Rianne M van der Linde*1, PhD; Professor Fiona E Matthews ${ }^{1,3}$; Professor Tom Dening ${ }^{2}$; Professor Carol Brayne ${ }^{1}$

*Corresponding author Institute of Public Health, University of Cambridge, United Kingdom

rmv23@medschl.cam.ac.uk

Department of Public Health and Primary Care

Herchel Smith Building

Forvie Site

Robinson Way

Cambridge CB2 OSR

+442082411169

1. Institute of Public Health, University of Cambridge, United Kingdom

2. Institute of Mental Health, University of Nottingham, United Kingdom

3. Institute of Health and Society, Newcastle University, United Kingdom

Conflicts of Interest and Source of Funding

No Disclosures to Report. RvdL received a studentship from National Institute for Health Research (NIHR) Collaborations for Leadership in Applied Health Research and Care (CLAHRC) for Cambridgeshire \& Peterborough.

Previous presentation

This work has been presented at the Alzheimer Association International Conference (AAIC), Boston 13-18 July 2013

Word count: 3,720 


\section{Tables and figures}

Figure 1: Transition between states

Figure 2: Flowchart of the presence of apathy over time

Table 1: One year transition rates taking into account age only

Table 2: One-year mortality risk of those with a symptom compared to those without

Table 3: Hazard ratio of the association between covariates and transition rates

Additional Tables and Figures to be published online:

Online Additional Figure 1: CFAS interviews

Online Additional Figure 2: full flowchart apathy that includes missing values

Online Additional Table 1: Baseline prevalence of symptoms, including how many are excluded

because single transition

Online Additional Table 2: Baseline characteristics

Online Additional Table 3: Symptom presence, missing values, loss to follow-up and drop out due to death across the follow-up period

Online Additional Table 4: Number of transitions for each of the symptoms

Online Additional Table 5 Hazard ratio of the association between symptom history and transition rates in those with at least 2 interviews

Online Additional Table 6 Sensitivity analysis comparing transition rates for an apathy definition based on participant interviews only to transition rates based on both participant and informant interviews 


\section{Abstract}

Objective To study the stability and emergence of a range of Behavioural and Psychological Symptoms (BPS), their association with mortality and the effect of covariates on these transitions in a population based study of cognitively impaired older people with a long follow-up period and large sample size, with a particular focus on apathy.

Methods Data were from a population-based, longitudinal cohort study of ageing. Interviews were conducted at $0,2,6,8$ and 10 years with 3,626 participants aged 65+. The persistence of 11 BPS and their association with mortality in those with cognitive impairment (MMSE 25 or below) was investigated using multistate models, allowing us to take into account estimations of the probability of transitions that occurred in the time between interviews.

Results Most BPS were persistent. Apathy was one of the most stable symptoms; in those with apathy, the probability of still having apathy after 1 year is 62\%. Apathy, sleep problems, depression, irritability and wandering were most likely to develop. BPS are associated with mortality; in those with apathy mortality is 3.1 times more likely than in those without apathy. Low cognitive function and dementia were associated with emergence of new symptoms.

Conclusions This population-based, multi-centre study with a follow-up period of 10 years showed that BPS are associated with mortality and most symptoms are persistent. Apathy was characterised by a high prevalence, a high persistence and a strong association with mortality, and has a negative impact on disability, management of other disease and caregiver burden. 


\section{Objective}

Behavioural and Psychological Symptoms (BPS) are present in over $80 \%$ of people with dementia (Garre-Olmo et al. 2010; Lyketsos et al. 2002; Steinberg et al. 2008; Zuidema et al. 2007), and are also common in those without dementia and in those with mild cognitive impairment (Monastero et al. 2009; Savva et al. 2009; van der Linde et al. 2010). In order to design and conduct clinical trials for the treatment of BPS, more information about the pattern of BPS over the course of dementia is needed to identify the best stage to intervene.

We have recently performed a systematic review of the longitudinal course of BPS (under review with the British Journal of Psychiatry). Most studies have recruited participants from psychiatric services, which have obvious sources of bias. Population-based studies were limited to looking at depression and most did not study the stability of symptoms, so data regarding the incidence and persistence of other common and important BPS, such as apathy, elation and wandering, are lacking.

This study overcomes many of the limitations of previous studies by studying a population-based sample (the Medical Research Council Cognitive Function and Ageing Study, MRC-CFAS) of over 3,500 participants aged 65 years and over with a prospective follow-up of 10 years. Eleven symptoms were studied: apathy, depression, anxiety, irritability, agitation, hallucination, persecution, misidentification, sleep problems, wandering and elation. Using a multistate model makes it possible to take into account estimations of the probability of transitions that occurred in the time between interviews.

Although, conventionally, BPS are just studied in the context of dementia, it is important to consider that some BPS may appear before the onset of or the diagnosis of dementia, and therefore the continuities 'before' and 'after' dementia are important to study. BPS in a cognitively healthy older person may indicate early dementia, and certain BPS, for example, depression, may indeed be risk factors for dementia. Common biological and psychosocial risk factors for BPS may exist among the cognitively healthy and cognitively impaired older populations. Therefore, we included all participants with cognitive impairments (MMSE<25) with or without dementia. 
We aimed to study the stability and emergence of a range of BPS, the association of BPS with mortality and the effect of covariates on these transitions in cognitively impaired older people.

\section{Methods}

\section{Sample}

Data were from the Medical Research Council Cognitive Function and Ageing Study (MRC CFAS), a multicentre longitudinal population-based study of ageing (Brayne et al. 2006). Participants aged 65 years and over were randomly selected from the Family Health Service Authority lists in two rural centres in Cambridgeshire and Gwynedd, and four urban centres in Liverpool, Newcastle, Nottingham and Oxford in the UK.

Screening interviews were conducted at baseline and two years. Individuals were randomly sampled into assessment interviews at both baseline and two years. The assessment interviews with participants and informal caregivers were completed at baseline and after 2, 6, 8 and 10 years follow-up as shown in Online Additional Figure 1. As some participants entered the study at one of the later interviews (due to the sampling into assessment interviews) and as in Liverpool (Saunders et al. 1993) an additional interview was conducted 2 years before the study started in the other 5 centres, the interviews are described as 'first interview', 'second interview', 'third interview', 'fourth interview', and 'fifth interview'.

Participants with a baseline Mini Mental State Examination (MMSE) score of 26 or higher $(n=2,134)$ were excluded from analyses, as well as participants with a single interview only for whom therefore no transitions were recorded (shown in Online Additional Table 1, for apathy: $n=72$ ).

\section{BPS and covariates}

Apathy, depression, anxiety, irritability, agitation, hallucination, persecution, misidentification, sleep problems, wandering and elation were measured using the Geriatric Mental State (GMS) Automated Geriatric Examination for Computer-Assisted 
Taxonomy (AGECAT) and Cambridge Mental Disorders of the Elderly Examination (CAMDEX) interviews with participants and informants as previously described by Savva et al. (Savva et al. 2009).

It has been previously shown that age, sex, cognitive function and dementia are associated with BPS and mortality and these variables were taken into account as covariates. Age was recorded at each interview. Only baseline cognitive function and dementia status were taken into account, as time varying covariates require a much more complex model with a much larger dataset. Baseline cognitive function was measured with the Mini Mental State Examination (MMSE). Scores were categorised in low (MMSE 0-18), moderate (MMSE 19-22) and high (MMSE 23-25) using previously defined cut-off scores (Huppert et al. 2005; Matthews et al. 2008; Stephan et al. 2010). Dementia status was derived using the full GMS-AGECAT diagnostic algorithm (Copeland et al. 1986).

BPS information at previous interviews was combined in a measure of BPS history. Reporting presence of BPS at one or more of the previous interviews was compared to never reporting BPS at the previous interviews. BPS history was not available at baseline.

\section{Mortality}

Date of death until 31 December 2006 was recorded for all participants based on information from the Office for National Statistics National Health Service Central Register. Participants who were alive at this time were censored, i.e. they are known to be alive but in an unknown state.

\section{Statistical methods}

The stability of BPS and their association with mortality was investigated using multi-state models. A multi-state Markov model is a way of describing the process in which an individual moves through a series of states in continuous time. It models death, decline and recovery within the same model and allows covariate effects to vary for each transition. The transitions between the following three states were analysed: "no symptom" (state 1), "symptom" (state 2) and "death" (state 3), as shown in Figure 1. Participants without symptoms could develop symptoms (transition from "no symptom" to "symptom", p12) or remain without symptoms (transition from "no symptom" to "no 
symptom", p11). Participants with symptoms could recover from their symptom (transition from "symptom" to "no symptom", p21) or symptoms could persist (transition from "symptom" to "symptom", p22). In addition, transitions to the absorbing death state are possible from the "symptom" state as well as the "no symptom" state. The use of history of the BPS in the model relaxes the first order Markov assumption and means the model is a semi-Markov model.

The maximum likelihood estimates and transition rates with 95\% confidence intervals were calculated. Age at the time of interview was used as the time variable and was therefore taken into account in all models. Hazard ratios of the association between covariates (sex, baseline MMSE and baseline dementia) and the transitions between states were calculated. In a semi-Markov model, the association between BPS history and transition between states was analysed in those with two or more interviews. A sensitivity analysis was performed using BPS definitions based on participant information only. Data cleaning and descriptive analyses were completed using Stata 12.0, followed by analysis using R version 2.15.3 (R Development Core Team 2010) and the "msm" package in $\mathrm{R}$ (Jackson 2011).

[Figure 1]

\section{Missing data}

Interviews where the BPS symptom sections were not administered were excluded from analysis. Other interviews for these participants were included in the analysis, except when excluding the interview with missing data resulted in the participant having only a single interview, in which case the participant was excluded.

\section{Results}

\section{Baseline characteristics}

At baseline, 3,626 participants were included. The mean age was 81.4 years, ranging from 65 to 105 , and approximately two thirds of the population were women. As we excluded 
those with a MMSE score above 25, all participants had cognitive impairment: $37 \%$ had low cognitive function (MMSE 0-18), 28\% moderate (MMSE 19-22) and 35\% high (MMSE $23-25)$. In addition, $37 \%$ had dementia at baseline (62\% of those with MMSE $0-18,16 \%$ of those with MMSE 19-22, and 5\% of those with MMSE 23-25).

The prevalence of BPS at the first interview with dementia where symptom presence is not missing is shown in Online Additional Table 1 and 2. More details on the prevalence of BPS in MRC-CFAS have been previously reported in those with and without dementia (Savva et al. 2009; van der Linde et al. 2010). Sleep problems and apathy have the highest prevalence and are seen in $43.1 \%$ and $31.9 \%$ of the population at baseline respectively. Irritability was also common (baseline prevalence $21.6 \%$ ), whereas psychotic symptoms were less often seen (baseline prevalence hallucination 9.2\%).

\section{Flowchart of symptoms over time}

Online Additional Table 3 shows the presence of symptoms, the number of missing values, loss to follow-up and drop out due to death across the interviews. Figure 2 shows the simplified flow chart of the presence of apathy over the 10 year follow-up period. The full flowchart that includes the number of missing values over time is available online (Online Additional Figure 2). Apathy was selected as an example because of its high prevalence (31.9\%, see Online Additional Table 1 ) which is consistent with findings in the literature that suggest it has a high incidence and prevalence, although its longitudinal course has not been studied frequently (systematic review under review with the British Journal of Psychiatry). The number of transitions for each of the symptoms is available online (Online Additional Table 4).

The figure suggests that apathy is stable. Participants with apathy seem more likely to report apathy again at the next interview than the people without apathy. For example, of the people with apathy at the first interview, 189 (16.7\%) report apathy at the next interview, compared to 155 (13.7\%) who do not. Of those without apathy at the first interview, 813 (33.6\%) do not report apathy at the next interview, compared to 244 (10.1\%) who do report apathy. During follow-up, 3,162 participants died and 392 were lost to follow-up. Those who died seem more likely to have reported apathy at the last 
interview before death. Across all interviews, 1,235 (67.7\%) of the people who reported apathy had died at the next interview, compared to 1,927 (50.4\%) of those who did not report apathy.

[Figure 2]

\section{One year transition rates}

Table 1 shows the estimated transition rates between the three states "no symptom" (state 1), "symptom" (state 2) and "death" (state 3) within one year. For example, a typical person without apathy had a probability of $13.5 \%$ of having developed apathy one year later, a probability of $76.3 \%$ of still not having apathy, and a probability of $10.2 \%$ of dying. A typical person with apathy had a probability of $62.2 \%$ of still having apathy one year later, a probability of $14.2 \%$ of recovering from apathy, and a probability of $23.6 \%$ of dying. Age is used as the time variable in the model and is the only covariate taken into account.

[Table 1]

\section{New symptoms}

Transitions from the "no symptom" state to the "symptom" state were most likely for apathy. The probability of developing apathy was $13.5 \%$. The transition rates for emergence of new symptoms generally reflect the prevalence of symptoms, with symptoms with a high prevalence such as apathy, sleep problems and depression showing high transition rates, and symptoms with a lower prevalence such as anxiety, hallucination and elation showing low transition rates.

\section{Stability of symptoms}

Apathy was one of the most stable symptoms (i.e. a high probability of staying in states "symptom" to "symptom"). In those with apathy, the probability of still having apathy after 1 year is $62 \%$. For most other symptoms the one year stability ranges between $35 \%$ and $45 \%$, while persecution, sleep problems and elation are not as stable. Wandering and misidentification also show a high stability. 


\section{Mortality}

For all symptoms except sleep problems the probability of dying is higher in those with the symptom than in those without. Table 2 shows the hazard ratios of the one-year transitions to death for those with the symptom compared to those without. Those with apathy or misidentification showed the highest mortality risk. Mortality is 3.1 times more likely in those with apathy than in those without apathy.

\section{[Table 2]}

\section{Association between covariates and the transition ratios}

Table 3 gives the estimated hazard ratios for the association between covariates and the transition rates.

\section{Sex}

Sex is not associated with symptom emergence or recovery, except for misidentification, where women are more likely to develop the symptom. For all symptoms there was a suggestion of a lower mortality risk for women mimicking the non symptom difference.

\section{Baseline cognitive function}

Some symptoms appear to be more likely to develop in those with low cognitive function compared to those with moderate cognitive function, including apathy, irritability, agitation, hallucination, persecution, misidentification and elation. Other symptoms, including depression, anxiety and wandering appear to be less likely to develop in those with low cognitive function. Significance is only reached for apathy and misidentification. For all symptoms, lower cognitive function is associated with mortality.

\section{Dementia at baseline}

All symptoms except anxiety and wandering are more likely to develop in people with baseline dementia. Some symptoms appeared to be more stable in dementia and have a lower recovery rate, including irritability, persecution, misidentification and elation, although these results are only significant for irritability. For most symptoms there is some evidence of a higher mortality risk for those with dementia. 
[Table 3]

\section{Symptom history}

Online Additional Table 5 shows the results of a semi-Markov model taking into account symptom history for participants with at least two interviews. All symptoms are more likely to develop in those who have reported the symptom in previous interviews. For some symptoms, including apathy, hallucination and elation, there also appears to be an increased mortality risk in those who did not report the symptom at the last interview before death, but who have reported the symptom in previous interviews.

\section{Sensitivity analysis}

A sensitivity analysis was performed using BPS definitions based on participant interviews only. Participant interviews were conducted on a larger number of participants than those with additional informant interviews and therefore larger numbers are available $(n=5,235$ compared to $n=3,626)$. The overall results and the association with mortality are similar to the results using both participant and informant information, although the prevalence, emergence of new symptoms and symptom stability are lower as the participant definitions are based on less information (Online Additional Table 6).

\section{Discussion}

\section{Summary of findings}

This study is important because it presents data from a large population-based sample with follow-up over a decade. The analyses presented here show that most BPS were persistent (wandering, apathy, misidentification, depression and anxiety showed the highest persistence), that some symptoms were more likely to develop than others (apathy, sleep problems, depression, irritability and wandering were most likely to develop), and that BPS are associated with mortality. Low cognitive function and dementia were associated with emergence of new symptoms. 


\section{Apathy}

Apathy showed a high prevalence, a high persistence (62\%), a high emergence of new symptoms (14\%) and a strong association with mortality. This is in agreement with the literature, although only a limited number of studies are available (Aalten et al. 2005; Wetzels et al. 2010).

Apathy has been defined as a disorder of motivation (Marin 1991; Robert et al. 2009; Starkstein et al. 2001) and is evidenced by diminished goal-directed overt behaviour, diminished goal-directed cognition, and diminished emotional concomitants of goaldirected behaviour. Here, apathy is defined using participant-based questions on slowing of thinking and talking, being less decisive and loss of energy and interest, and informantbased questions on loss of energy and interest and giving up a special skill or hobby (Savva et al. 2009) While less detail is included than in apathy-specific instruments (e.g. Apathy Evaluation Scale), the measurement of apathy used here includes questions of all three domains of the apathy definition by Marin et al. The Neuropsychiatric Inventory (Cummings et al. 1994), the most commonly used instrument to measure BPS, defines apathy as a loss of interest. Loss of interest was also included in the definition used here, but we also included questions on the slowing of thinking and talking and being less decisive which are not included in the NPI.

Symptoms of apathy and depression overlap and the two disorders can be difficult to distinguish. Apathy and depression frequently occur together, but both can occur in absence of the other. The issue is still under debate, but mostly apathy is considered a separate syndrome (Starkstein et al. 2005; Tagariello et al. 2009). The different pattern and stability of apathy and depression found here support the view that apathy is a separate syndrome.

The high prevalence and high stability of apathy and its associations with disability, poorer health and caregiver stress mean that apathy can be a large burden. Apathy is associated with disability (Benoit et al. 2008; Freels et al. 1992; Starkstein et al. 2006) and people with apathy may rely on others to initiate behaviour even when they are still capable of performing the activity (Landes et al. 2001). Apathy is associated with poorer physical health, self-neglect, poor nutrition and poor medication adherence and can 
complicate the management of other conditions including dementia (Benoit et al. 2008), diabetes (Padala et al. 2008) and cardiovascular disease (Mayo et al. 2009; Starkstein et al. 1993). In addition, apathy can increase caregiver burden (Hart et al. 2003; Kaufer et al. 1998; Pang et al. 2002; Thomas et al. 2001). Apathy may also reduce quality of life of the patient (Samus et al. 2005); although it has been suggested that apathy may not be a concern to the patient themselves and has been associated with lack of awareness in dementia (Aalten et al. 2006; Derouesne et al. 1999).

All BPS were associated with mortality. The strongest association was found for apathy ( $\mathrm{HR}=3.1,95 \% \mathrm{Cl}$ 2.7-3.7) because of the low risk of death in those without apathy (10.2\% compared to $13.2-17.7 \%$ for the other symptoms). This low risk of death in those without apathy might be explained by the high prevalence and stability of apathy. Participants are less likely to have one of the less prevalent symptoms before death. Apathy might be associated with mortality because of its associations with physical health and self-neglect as described in the previous paragraph, or those with apathy or other BPS may have certain neurobiological changes associated with mortality. Conversely, people who lack apathy are likely to be physically and socially more active. They are presumably more motivated to take care of themselves, eat well and comply with prescribed medication. This is likely to be associated with better health and lower mortality.

Apathy may often be overlooked clinically because unlike other symptoms, such as irritability, it is less obtrusive to carers and professional staff. However, its association with a range of poor outcomes suggests that it should be a more active target for therapy and research. For example, the alerting properties of cholinesterase inhibitors may be helpful, or perhaps interventions aimed at increasing physical activity and cognitive stimulation should be encouraged.

\section{Strengths and limitations}

The main strengths of this study are the population-based recruitment of participants, the large sample size and the long (10 years) follow-up period. Eleven BPS were studied, including apathy and elation. Sensitivity analyses showed similar results and the model reproduced well known associations between male sex and mortality and between lower cognitive function and mortality. 
This study adds to the literature by studying the symptoms over a longer time period, measuring BPS every two or four years. Using a multistate model made it possible to take into account estimations of the probability of transitions that occurred in the time between interviews.

BPS history was not measured at baseline and the association between BPS history and transition rates could only be studied in those with at least two interviews.

As in all longitudinal studies, loss to follow-up is a challenge. It has previously been shown that individuals who refused to participate in the 2 year follow-up interview were more likely to have poorer cognitive function, to have less education, and to live in alone in one of the rural centres. Those who moved away or were uncontactable were more likely to be single, smokers or depressed or to have dementia (Matthews et al. 2004). As several of these factors have been associated with presence of BPS and mortality, results might have been different if these participants could have been included in the analyses.

It would have been interesting to compare our results in those with a MMSE score below 25 to those with a diagnosis of dementia. However, this was not possible because of the relatively low number of people with dementia and their reduced survival. In addition, cognitive function severity bands (MMSE 0-18, 19-22 and 23-25) were based on previous analysis in MRC CFAS that compared the predictive probability of each MMSE score to detect those who develop dementia in 2 years (Huppert et al. 2005; Matthews et al. 2008; Stephan et al. 2010). Such groups, based on cognitive test scores, are not perfectly matched to the detection of dementia, though obviously the lower band of MMSE scores is likely to contain a high proportion of people with dementia. Furthermore, the difference between the low MMSE group (upper band: 18) and the high MMSE group (lower band: 23) may in some cases be as little as 5 points. It may also be that the persistence of BPS differs between these MMSE groups, particularly in the most severely impaired (MMSE $0-18$ ) and in those with dementia (37\% of the study population). It should also be borne in mind that a MMSE score below 25 does not necessarily imply that the individual has cognitive impairment and some individuals may have been misclassified. 
We cannot exclude residual confounding. Age has been taken into account in all models, and the associations with baseline cognitive function, baseline dementia and BPS history have been investigated. Dementia and cognitive function were taken into account at baseline only, as using covariate information from all interviews would make the model too complex. A range of other factors have been associated with BPS, cognitive function and mortality and may have confounded the results, including disability, physical health, education, social class, social relationships, treatment and antipsychotic use and comorbidity.

\section{Conclusion}

This population-based, multi-centre study with a follow-up period of 10 years showed that BPS are associated with mortality and most symptoms were persistent. Apathy was characterised by a high prevalence, a high persistence and a strong association with mortality, and it has a negative impact on disability, management of other diseases and caregiver burden. 


\section{References}

Aalten P, de Vugt ME, Jaspers N, Jolles J, Verhey FR. 2005. The course of neuropsychiatric symptoms in dementia. Part I: findings from the two-year longitudinal Maasbed study. 20(6): 523-530.

Aalten P, van VE, de Vugt ME, Lousberg R, Jolles J, Verhey FR. 2006. Awareness and behavioral problems in dementia patients: a prospective study. 18(1): 3-17.

Benoit M, Andrieu S, Lechowski L, Gillette-Guyonnet S, Robert PH, Vellas B. 2008. Apathy and depression in Alzheimer's disease are associated with functional deficit and psychotropic prescription. 23(4): 409-414.

Brayne C, McCracken C, Matthews FE. 2006. Cohort profile: the Medical Research Council Cognitive Function and Ageing Study (CFAS). International Journal of Epidemiology 35(5): 1140-1145.

Copeland JR, Dewey ME, Griffiths-Jones HM. 1986. A computerized psychiatric diagnostic system and case nomenclature for elderly subjects: GMS and AGECAT. Psychologie Medicale 16(1): 8999.

Cummings JL, Mega M, Gray K, Rosenberg-Thompson S, Carusi DA, Gornbein J. 1994. The Neuropsychiatric Inventory: comprehensive assessment of psychopathology in dementia. Neurology 44(12): 2308-2314.

Derouesne C, Thibault S, Lagha-Pierucci S, Baudouin-Madec V, Ancri D, Lacomblez L. 1999. Decreased awareness of cognitive deficits in patients with mild dementia of the Alzheimer type. 14(12): 1019-1030.

Freels S, Cohen D, Eisdorfer C, Paveza G, Gorelick P, Luchins DJ, Hirschman R, Ashford JW, Levy P, Semla $T,$. 1992. Functional status and clinical findings in patients with Alzheimer's disease. 47(6): M177-M182.

Garre-Olmo J, Lopez-Pousa S, Vilalta-Franch J, de Gracia BM, Vilarrasa AB. 2010. Grouping and trajectories of the neuropsychiatric symptoms in patients with Alzheimer's disease, part I: symptom clusters. 22(4): 1157-1167.

Hart DJ, Craig D, Compton SA, Critchlow S, Kerrigan BM, Mcllroy SP, Passmore AP. 2003. A retrospective study of the behavioural and psychological symptoms of mid and late phase Alzheimer's disease. 18(11): 1037-1042.

Huppert FA, Cabelli ST, Matthews FE. 2005. Brief cognitive assessment in a UK population sample -distributional properties and the relationship between the MMSE and an extended mental state examination. 5: 7 .

Jackson C. 2011. Multi-State Models for Panel Data: The msm package for R. Journal of Statistical Software 38: 1-29.

Kaufer DI, Cummings JL, Christine D, Bray T, Castellon S, Masterman D, MacMillan A, Ketchel P, DeKosky ST. 1998. Assessing the impact of neuropsychiatric symptoms in Alzheimer's disease: the Neuropsychiatric Inventory Caregiver Distress Scale. Journal of the American Geriatrics Society 46(2): 210-215.

Landes AM, Sperry SD, Strauss ME, Geldmacher DS. 2001. Apathy in Alzheimer's disease. Journal of the American Geriatrics Society 49(12): 1700-1707.

Lyketsos CG, Lopez O, Jones B, Fitzpatrick AL, Breitner J, DeKosky S. 2002. Prevalence of neuropsychiatric symptoms in dementia and mild cognitive impairment: results from the cardiovascular health study. 288(12): 1475-1483.

Marin RS. 1991. Apathy: a neuropsychiatric syndrome. 3(3): 243-254.

Matthews FE, Chatfield M, Freeman C, McCracken C, Brayne C. 2004. Attrition and bias in the MRC cognitive function and ageing study: an epidemiological investigation. 4: 12 .

Matthews FE, Stephan BC, McKeith IG, Bond J, Brayne C. 2008. Two-year progression from mild cognitive impairment to dementia: to what extent do different definitions agree? Journal of the American Geriatrics Society 56(8): 1424-1433.

Mayo NE, Fellows LK, Scott SC, Cameron J, Wood-Dauphinee S. 2009. A longitudinal view of apathy and its impact after stroke. 40(10): 3299-3307. 
Monastero R, Mangialasche F, Camarda C, Ercolani S, Camarda R. 2009. A systematic review of neuropsychiatric symptoms in mild cognitive impairment. Journal of Alzheimer's Disease 18(1): 11-30.

Padala PR, Desouza CV, Almeida S, Shivaswamy V, Ariyarathna K, Rouse L, Burke WJ, Petty F. 2008. The impact of apathy on glycemic control in diabetes: a cross-sectional study. 79(1): 37-41.

Pang FC, Chow TW, Cummings JL, Leung VP, Chiu HF, Lam LC, Chen QL, Tai CT, Chen LW, Wang SJ, Fuh JL. 2002. Effect of neuropsychiatric symptoms of Alzheimer's disease on Chinese and American caregivers. 17(1): 29-34.

R Development Core Team. 2010. R: A language and environment for statistical computing. In Foundation for Statistical Computing: Vienna, Austria.

Robert P, Onyike CU, Leentjens AF, Dujardin K, Aalten P, Starkstein S, Verhey FR, Yessavage J, Clement JP, Drapier D, Bayle F, Benoit M, Boyer P, Lorca PM, Thibaut F, Gauthier S, Grossberg G, Vellas B, Byrne J. 2009. Proposed diagnostic criteria for apathy in Alzheimer's disease and other neuropsychiatric disorders. 24(2): 98-104.

Samus QM, Rosenblatt A, Steele C, Baker A, Harper M, Brandt J, Mayer L, Rabins PV, Lyketsos CG. 2005. The association of neuropsychiatric symptoms and environment with quality of life in assisted living residents with dementia. 45 Spec No 1(1): 19-26.

Saunders PA, Copeland JR, Dewey ME, Gilmore C, Larkin BA, Phaterpekar H, Scott A. 1993. The prevalence of dementia, depression and neurosis in later life: the Liverpool MRC-ALPHA Study. 22(5): 838-847.

Savva GM, Zaccai J, Matthews FE, Davidson JE, McKeith I, Brayne C. 2009. Prevalence, correlates and course of behavioural and psychological symptoms of dementia in the population. British Journal of Psychiatry 194(3): 212-219.

Starkstein SE, Fedoroff JP, Price TR, Leiguarda R, Robinson RG. 1993. Apathy following cerebrovascular lesions. 24(11): 1625-1630.

Starkstein SE, Ingram L, Garau ML, Mizrahi R. 2005. On the overlap between apathy and depression in dementia. 76(8): 1070-1074.

Starkstein SE, Jorge R, Mizrahi R, Robinson RG. 2006. A prospective longitudinal study of apathy in Alzheimer's disease. 77(1): 8-11.

Starkstein SE, Petracca G, Chemerinski E, Kremer J. 2001. Syndromic validity of apathy in Alzheimer's disease. 158(6): 872-877.

Steinberg M, Shao H, Zandi P, Lyketsos CG, Welsh-Bohmer KA, Norton MC, Breitner JC, Steffens DC, Tschanz JT. 2008. Point and 5-year period prevalence of neuropsychiatric symptoms in dementia: the Cache County Study. 23(2): 170-177.

Stephan BC, Savva GM, Brayne C, Bond J, McKeith IG, Matthews FE. 2010. Optimizing mild cognitive impairment for discriminating dementia risk in the general older population. American Journal of Geriatric Psychiatry 18(8): 662-673.

Tagariello P, Girardi P, Amore M. 2009. Depression and apathy in dementia: same syndrome or different constructs? A critical review. 49(2): 246-249.

Thomas P, Clement JP, Hazif-Thomas C, Leger JM. 2001. Family, Alzheimer's disease and negative symptoms. 16(2): 192-202.

van der Linde R, Stephan BC, Matthews FE, Brayne C, Savva GM. 2010. Behavioural and psychological symptoms in the older population without dementia - relationship with socio-demographics, health and cognition. 10(1): 87.

Wetzels RB, Zuidema SU, de Jonghe JF, Verhey FR, Koopmans RT. 2010. Course of neuropsychiatric symptoms in residents with dementia in nursing homes over 2-year period. American Journal of Geriatric Psychiatry 18(12): 1054-1065.

Zuidema SU, Derksen E, Verhey FR, Koopmans RT. 2007. Prevalence of neuropsychiatric symptoms in a large sample of Dutch nursing home patients with dementia. 22(7): 632-638. 


\section{Figures}

Figure 1 Transition between states

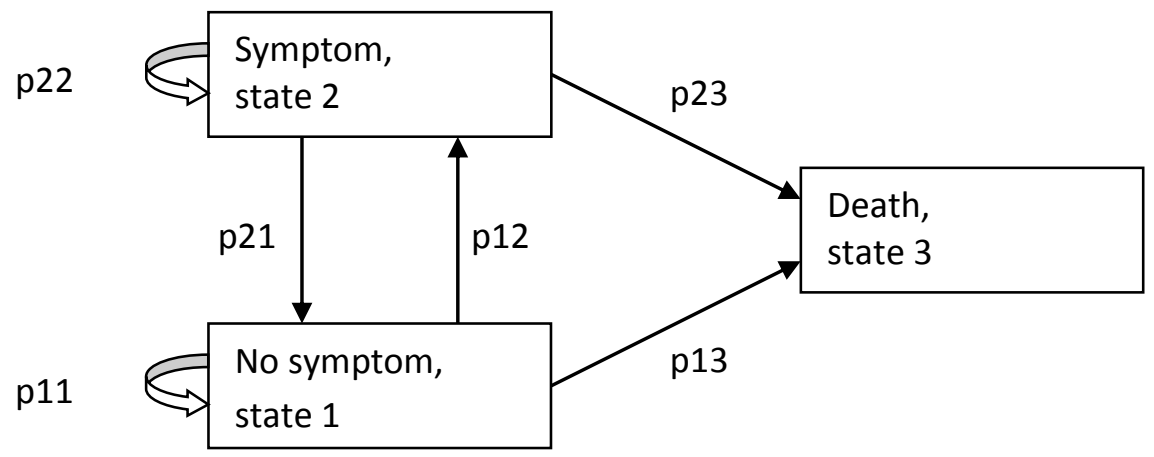


Figure 2 Flowchart of the presence of apathy over time

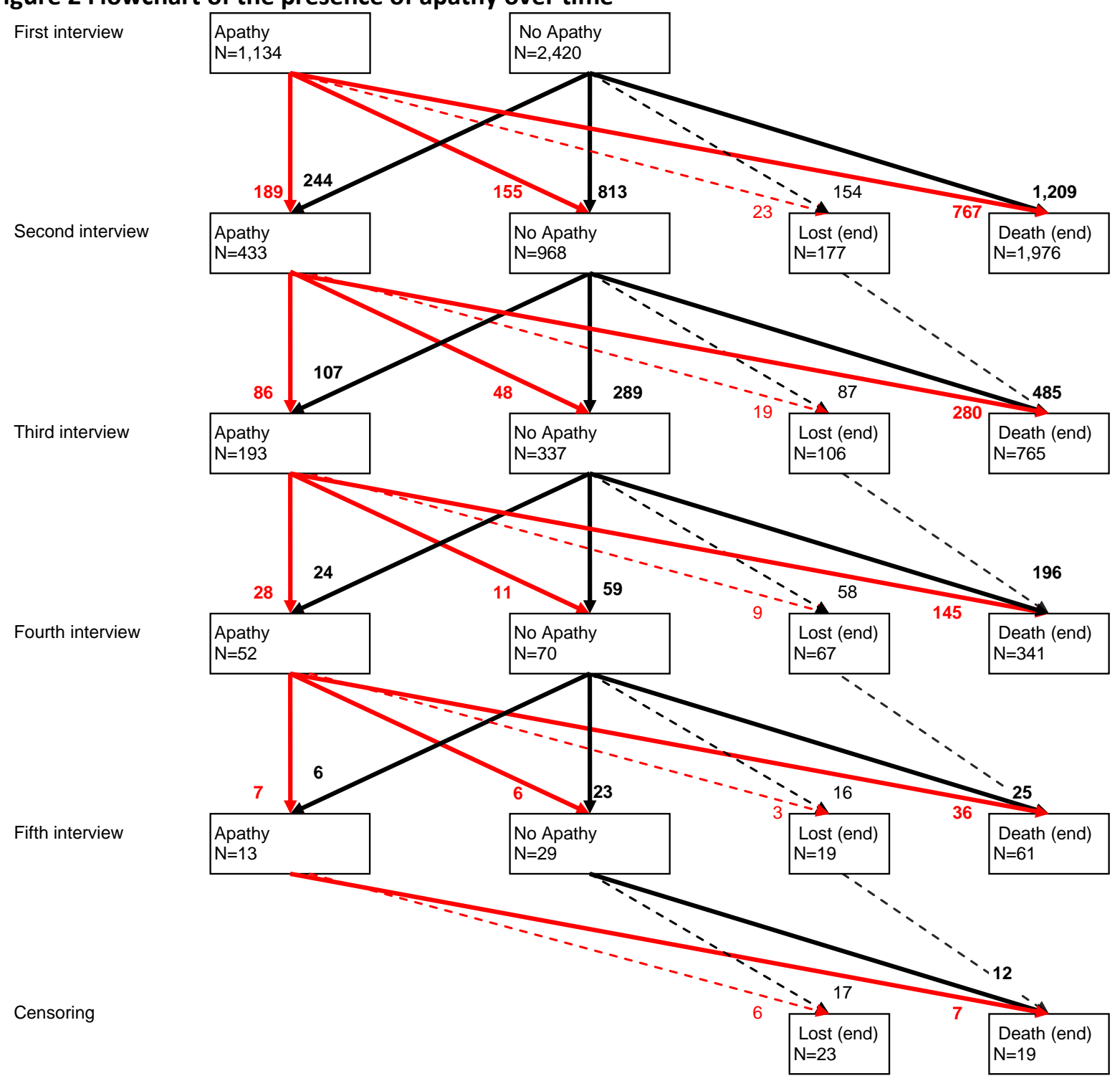




\section{Tables}

Table 1 One year transition rates taking into account age only

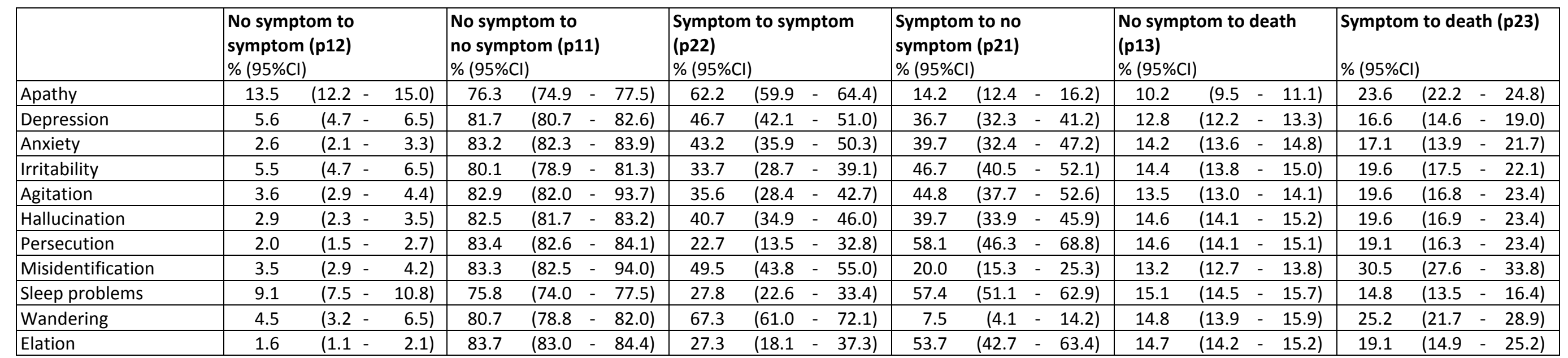

$\mathrm{Cl}$, confidence interval

Age is used as the time variable in the model and is the only covariate taken into account. Please see Table 3 for the hazard ratio of the association between covariates and transition rates

Table 2 One-year mortality risk of those with a symptom compared to those without

\begin{tabular}{|l|l|}
\hline & $\begin{array}{l}\text { Estimation of the ratio of the mortality } \\
\text { rates for those with the symptom and } \\
\text { those without the symptom } \\
\text { Hazard ratio }(95 \% \mathrm{Cl})\end{array}$ \\
\hline Apathy & \multicolumn{1}{|c|}{$3.1(2.7-3.7)$} \\
\hline Depression & $1.5(1.2-1.8)$ \\
\hline Anxiety & $1.3(0.9-1.9)$ \\
\hline Irritability & $1.7(1.4-2.1)$ \\
\hline Agitation & $1.8(1.4-2.3)$ \\
\hline Hallucination & $1.5(1.2-2.0)$ \\
\hline
\end{tabular}




\begin{tabular}{|l|l|}
\hline Persecution & $1.6(1.2-2.2)$ \\
\hline Misidentification & $3.0(2.5-3.5)$ \\
\hline Sleep problems & $1.0(0.8-1.2)$ \\
\hline Wandering & $1.9(1.5-2.4)$ \\
\hline Elation & $1.6(1.0-2.4)$ \\
\hline
\end{tabular}

$\mathrm{Cl}$, confidence interval

Age is used as the time variable in the model and is the only covariate taken into account. Please see Table 3 for the hazard ratio of the association between covariates and transition rates 
Table 3 Hazard ratio of the association between covariates and transition rates

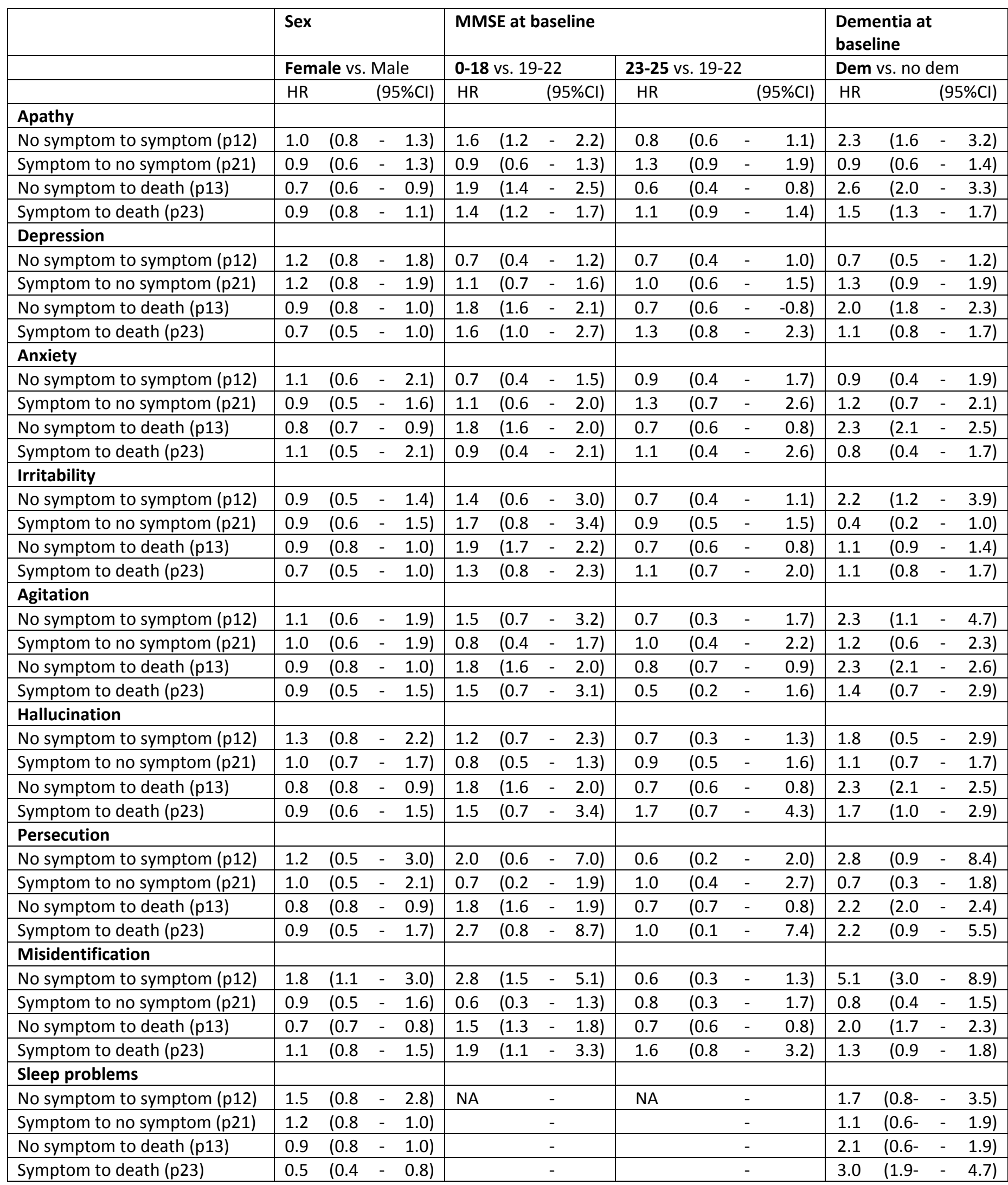


Table 3 continued

\begin{tabular}{|c|c|c|c|c|c|c|c|c|c|c|c|c|c|c|c|c|}
\hline \multicolumn{17}{|l|}{ Wandering } \\
\hline No symptom to symptom (p12) & 1.0 & $(0.4$ & - & 2.3) & 0.6 & 10.2 & - & 1.7) & 0.9 & 10.3 & - & $2.4)$ & 0.6 & 10.0 & - & 7.2) \\
\hline Symptom to no symptom (p21) & 0.9 & $(0.2$ & - & 4.1) & 1.1 & $(0.2$ & - & 6.3) & 0.7 & $(0.1$ & - & 6.7) & 1.2 & 10.0 & - & 27.8) \\
\hline No symptom to death (p13) & 0.8 & $(0.7$ & - & 1.0) & 2.1 & $(1.5$ & 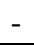 & 2.8) & 0.6 & 10.3 & - & 1.1) & 1.5 & $(1.0$ & - & 2.1) \\
\hline Symptom to death (p23) & 0.9 & $(0.6$ & - & 1.4) & 1.0 & (0.6 & - & 1.6) & 1.2 & $(0.6$ & 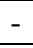 & 2.2) & 0.7 & $(0.3$ & - & 1.7) \\
\hline \multicolumn{17}{|l|}{ Elation } \\
\hline No symptom to symptom (p12) & 1.2 & $(0.4$ & - & 3.3) & 2.2 & $(0.8$ & - & $6.1)$ & 0.5 & $(0.2$ & - & 1.4) & 2.7 & $(1.1$ & - & 6.4) \\
\hline Symptom to no symptom (p21) & 0.6 & $(0.3$ & - & 1.4) & 1.2 & $(0.5$ & - & 2.9) & 0.9 & $(0.4$ & - & 2.0) & 0.8 & $(0.4$ & - & 1.6) \\
\hline No symptom to death (p13) & 0.9 & 10.8 & - & 1.0) & 1.8 & $(1.6$ & - & $2.0)$ & 0.7 & $(0.7$ & - & $0.8)$ & 2.2 & $(2.2$ & - & $2.4)$ \\
\hline Symptom to death (p23) & 0.6 & $(0.2$ & - & 1.4) & 4.4 & 10.6 & - & 29.2) & 0.4 & 10.0 & - & 13.3) & 3.0 & 13.0 & - & 9.8) \\
\hline
\end{tabular}

NA Too few transitions to model, model does not converge

$\mathrm{Cl}$, confidence interval

MMSE, mini mental state examination

Dem, dementia 